\title{
Hyperglycemia After Metformin Overdose: A Case Report
}

\author{
Sabiha Sahin ${ }^{\mathrm{a}, \mathrm{d}}$, Cigdem Binay ${ }^{\mathrm{b}}$, Enver Simsek ${ }^{\mathrm{b}}$, Ener Cagri Dinleyici ${ }^{\mathrm{a}}$, \\ Kursat Bora Carman ${ }^{\mathrm{c}}$
}

\begin{abstract}
We present a case of a 16-year-old non-diabetic girl who ingested over 35 tablets (glucophage Merck $850 \mathrm{mg}$ ) of metformin in a suicide attempt. She presented to pediatric emergency department with severe lactic acidosis and a progressively increasing serum glucose level. She was in a coma state at the time of admission, Glasgow coma scale was 3/15 and arterial blood pressure was 106/45 mm Hg. Arterial blood gas (ABG) analysis indicated severe metabolic acidosis ( $\mathrm{pH}$ 6.7) with high anion gap $-30.5, \mathrm{PCO}_{2} 13.2, \mathrm{HCO}_{3} 3.9, \mathrm{BE}$ -30 , lactate $14.54 \mathrm{mmol} / \mathrm{L}$, blood glucose 497 , amylase $531 \mathrm{IU} / \mathrm{L}$, and uric acid $10.47 \mathrm{mg} / \mathrm{dL}$. Serum ethanol, acetaminophen and salicylates were measured and found to be undetectable. Electrocardiographic monitoring demonstrated a narrow-complex sinus tachycardia. She was intubated, totally $2,000 \mathrm{cc} / \mathrm{m}^{2}$ fluid and $\mathrm{NaHCO}_{3}$ were given, and insulin infusion 0.1 units $/ \mathrm{kg}$ was started for blood glucose of 497 $\mathrm{mg} / \mathrm{dL}$. But the patient suffered several cardiac arrests with pulseless electrical activity and ultimately expired $25 \mathrm{~h}$ after the ingestion. The patient was transferred to pediatric intensive care unit (PICU) for high-volume continuous veno-venous hemofiltration (CVVH). Despite the supportive care in ICU, she died due to multiple organ failures after $48 \mathrm{~h}$ of hospitalization.
\end{abstract}

Keywords: Lactic acidosis; Metformin poisoning; Pancreatitis; Hyperglycemia

\section{Introduction}

Metformin is a biguanide anti-hyperglycemic drug which is the most commonly prescribed oral agent to treat diabetes mellitus. Metformin-associated lactic acidosis (MALA) occurs infrequently with therapeutic use, occurring in 0.03 cases per

Manuscript accepted for publication November 01, 2016

aDivision of Paediatric Emergency and Critical Care, Department of Paediatrics, Osmangazi University School of Medicine, Eskisehir, Turkey

bDivision of Paediatric Endocrinology, Department of Paediatrics, Osmangazi University School of Medicine, Eskisehir, Turkey

'Division of Paediatric Neurology, Department of Paediatrics, Osmangazi University School of Medicine, Eskisehir, Turkey

${ }^{\mathrm{d} C}$ Corresponding Author: Sabiha Sahin, Division of Paediatric Emergency and Critical Care, Department of Paediatrics, Osmangazi University School of Medicine, Eskisehir, Turkey. Email: sabiha.sahin@mynet.com

doi: https://doi.org/10.14740/ijcp259w
1,000 patient-years [1]. However, in overdose situations, metformin toxicity is associated with development of lactic acidosis $[2,3]$. Because metformin does not induce insulin release by pancreatic beta-islet cells, exposures infrequently induce disorders of glucose homeostasis. When abnormal glucose levels occur with metformin exposures, hypoglycemia is much more commonly seen than hyperglycemia; hypoglycemia is usually ascribed to concurrent anti-diabetic medications such as the sulfonylureas. Hyperglycemia appears to be a marker of severe toxicity in cases of metformin poisoning, and may be associated with the patient's underlying diabetes or the development of pancreatitis [4]. We present the case of a 16-yearold non-diabetic girl who took a large overdose of metformin in a suicide attempt. She presented with severe lactic acidosis and a progressively increasing serum glucose level. The hyperglycemia might have been due to pancreatitis. The patient's peak serum glucose level of $497 \mathrm{mg} / \mathrm{dL}$ is the highest yet reported in a case of metformin toxicity in children.

\section{Case Report}

A 16-year-old female patient without history of any disease ingested metformin in a suicide attempt. The patient consumed metformin bottle that contained about 30 - 35 tablets of 850 $\mathrm{mg}$ each $4 \mathrm{~h}$ ago. After the activated charcoal and gastric decontamination was applied, because of the development of unconsciousness and high serum glucose $(497 \mathrm{mg} / \mathrm{dL})$ level, she was referred to our pediatric emergency department (PED) from an outer medical center. Vital signs on arrival to the PED were temperature of $35.2{ }^{\circ} \mathrm{C}$, pulse of 123 beats $/ \mathrm{min}$, blood pressure of $106 / 45 \mathrm{~mm} \mathrm{Hg}$, and respirations at 20 breaths/min on intubated. Glasgow coma scale score was 3 . The patient was hospitalized in pediatric intensive care unit (PICU). The laboratory examinations are shown in Table 1 . Serum toxicological analyze was negative for ethanol, tricyclic antidepressants, benzodiazepine, salicylate and paracetamol. Metformin level assessment is important in toxicity; however, this facility is not available in our hospital. Electrocardiographic (ECG) monitoring demonstrated a narrow-complex sinus tachycardia. Abdomen ultrasound was normal with no sign of any intraperitoneal collection or splenomegaly. By the laboratory results and history, the patient was diagnosed with acute metformin poisoning. $\mathrm{ABG}$ analysis showed persistent metabolic acidosis. Insulin perfusion 0.1 units $/ \mathrm{kg}$ was started for high serum glucose level. Dopamine drip infusion was started for hypotension despite continued IV hydration with $0.9 \%$ saline bolus. Hemodialysis was performed two times. Four hours af- 
Table 1. Laboratory Results of the Patient

\begin{tabular}{lll}
\hline & $\begin{array}{l}\text { Initial laboratory } \\
\text { evaluation (ED arrival) }\end{array}$ & After dialysis \\
\hline $\mathrm{pH}$ & 6.7 & 7.13 \\
$\mathrm{pCO}_{2}(\mathrm{~mm} \mathrm{Hg})$ & 13.2 & 36 \\
$\mathrm{HCO}_{3}(\mathrm{~mm} \mathrm{Hg})$ & 3.9 & 12.4 \\
$\mathrm{BE}(\mathrm{mmol} / \mathrm{L})$ & 30 & 17 \\
Anion gap & -30.5 & 17.4 \\
Lactate $(\mathrm{mmol} / \mathrm{L})$ & 14.54 & 13.6 \\
Glucose $(\mathrm{mg} / \mathrm{dL})$ & 497 & 378 \\
Serum amylase $(\mathrm{IU} / \mathrm{L})$ & 531 & 668 \\
\hline
\end{tabular}

ter PICU admission, she arrested in at several times and was resuscitated. After the second hemodialysis, laboratory examinations showed $\mathrm{pH} 7.13, \mathrm{pCO}_{2} 36 \mathrm{~mm} \mathrm{Hg}, \mathrm{pO}_{2} 114 \mathrm{~mm} \mathrm{Hg}$, bicarbonate $12.4 \mathrm{mEq} / \mathrm{L}, \mathrm{BE} 17$, and lactate $13.60 \mathrm{mmol} / \mathrm{L}$ (Table 1). Her serum glucose, lactate and serum amylase levels did not return to normal levels during the clinical observation. The patient died due to multiple organ failure $48 \mathrm{~h}$ after hospitalization despite the supportive care in PICU.

\section{Discussion}

Metformin is a biguanide anti-hyperglycemic drug which is the most commonly prescribed oral agent to treat diabetes mellitus. It reduces hepatic gluconeogenesis and enhances peripheral glucose utilization. Unlike sulfonylurea-based oral hypoglycemics, metformin does not promote weight gain. It may be helpful in the management of some forms of obesity and is used to promote fertility in polycystic ovary syndrome. The bioavailability of metformin is $40-60 \%$. Its peak plasma concentration is reached by $3.0 \pm 0.3 \mathrm{~h}$ and it is completely absorbed by $6 \mathrm{~h}$ after oral ingestion [4]. Overdoses with metformin are relatively uncommon, but may have serious consequences. In a 5-year review of toxic exposures reported to US Poison Control Centers, only 4,072 out of nearly 11 million exposures involved metformin, corresponding to less than 1 in 2,500 [5]. Lactic acidosis was seen in $1.6 \%$ of metformin exposures reported to poison control centers, but only $10 \%$ of these exposures were due to intentional overdoses [5]. Such publications, however, form an obviously biased group, and the actual incidence of lactic acidosis with intentional metformin overdose remains unknown [6]. Case reports and series of severe toxicity from metformin are typically associated with severe lactic acidosis, and many of these are due to intentional overdose. The prognosis in cases of undifferentiated lactic acidosis is poor, with an expected case-fatality rate of $30-50 \%$. This unusual finding suggests that patients developing lactic acidosis at lower metformin levels probably have concurrent underlying disease, placing them at higher risk both for accumulating lactate and for death. Lactic acidosis from metformin overdose is clinically indistinguishable from other causes. Because it is caused by impairing the liver's lactate clearance and not the direct result of tissue hypoxia. Lactic acidosis is a common etiology in patients presenting with an anion gap metabolic acidosis and metformin is among several toxicants well known to cause lactic acidosis [7].

We report the case of an intentional overdose of metformin in a pediatric patient without diabetes which resulted in progressive hyperglycemia early in the clinical course and fatal lactic acidosis. This patient's peak serum glucose level of 497 $\mathrm{mg} / \mathrm{dL}$ is the highest reported in a case of metformin toxicity in pediatric age group.

Metformin generally does not cause significant alterations in serum glucose levels, even in overdose situations. Although metformin does not directly lower glucose levels, metforminassociated hypoglycemia has been reported, both with therapeutic dosing and with overdoses.

When abnormal glucose levels occur with metformin exposures, hypoglycemia is much more commonly seen than hyperglycemia; hypoglycemia is usually ascribed to concurrent anti-diabetic medications such as the sulfonylureas [8].

Hyperglycemia has been related to acute pancreatitis in a few cases of metformin toxicity from both intentional overdose and therapeutic dosing [5]. Since our patient did not have a history of diabetes, extreme hyperglycemia of patient is more unexpected. With a lack of circulating insulin, metformin's ability to enhance peripheral insulin sensitivity would count from the acute physiologic stress of the overdose contributed to the hyperglycemia [9]. Alternate toxicologic causes for this patient's hyperglycemia seem unlikely. Only a few toxins are routinely associated with hyperglycemia, including calcium channel blockers and the ingestion of agents that specifically poison pancreatic beta-islet cells, such as alloxan, nitrophenolurea (an obsolete rodenticide) and streptozocin (a chemotherapy agent for pancreatic islet cell tumors). Calcium channel blockers can impair insulin release and often result in modest hyperglycemia. In our patient, there was no history for usage of these drugs and the patient did not present with hypotension or bradycardia.

Hyperglycemia appears to be a marker of severe toxicity in cases of metformin poisoning, and may be associated with the patient's underlying diabetes or the development of pancreatitis [10]. In our case, pancreatitis was diagnosed serologically with high serum amylase level. Another potential complicating factor was the elevated osmolal gap in our patient. An unexplained osmolal gap may be seen in several severe diseases, including shock or sepsis, and it was also seen in four out of nine cases of fatal metformin toxicity, providing an alternate explanation for its presence $[11,12]$. On the other hand, there was no historical evidence supporting the ingestion of any alcohol other than methanol, nor calcium oxalate crystalluria from ethylene glycol. Also, she had no ketonuria which would occur with isopropanol ingestion.

Metformin level assessment is important toxicity; however, this facility is not available in our hospital.

\section{Conclusion}

Metformin-induced pancreatitis has rarely been reported as direct complication. It has been found in the presence of renal failure or multiple drug ingestion. The progressive hyperglyce- 
mia early in the clinical course and fatal lactic acidosis should suggest acute pancreatitis related to metformin overdose [13]. This case is the first metformin intoxication published case in pediatric group with hyperglycemia and acute pancreatitis.

\section{References}

1. Bailey CJ, Turner RC. Metformin. N Engl J Med. 1996;334(9):574-579.

2. Wills BK, Bryant SM, Buckley P, Seo B. Can acute overdose of metformin lead to lactic acidosis? Am J Emerg Med. 2010;28(8):857-861.

3. Heaney D, Majid A, Junor B. Bicarbonate haemodialysis as a treatment of metformin overdose. Nephrol Dial Transplant. 1997;12(5):1046-1047.

4. Garber AJ. Metformin: mechanisms of antihyperglycemic action, other pharmacodynamic properties, and safety perspectives. Endocr Pract. 1997;3(6):359-370.

5. Spiller HA, Quadrani DA. Toxic effects from metformin exposure. Ann Pharmacother. 2004;38(5):776-780.

6. Chan NN, Brain HP, Feher MD. Metformin-associated lactic acidosis: a rare or very rare clinical entity? Diabet Med. 1999;16(4):273-281.
7. Lacher M, Hermanns-Clausen M, Haeffner K, Brandis M, Pohl M. Severe metformin intoxication with lactic acidosis in an adolescent. Eur J Pediatr. 2005;164(6):362365 .

8. Suchard JR, Grotsky TA. Fatal metformin overdose presenting with progressive hyperglycemia. West J Emerg Med. 2008;9(3):160-164.

9. von Mach MA, Sauer O, Sacha Weilemann L. Experiences of a poison center with metformin-associated lactic acidosis. Exp Clin Endocrinol Diabetes. 2004;112(4):187190.

10. Dell'Aglio DM, Perino LJ, Todino JD, Algren DA, Morgan BW. Metformin overdose with a resultant serum pH of 6.59: survival without sequalae. J Emerg Med. 2010;39(1):e77-80.

11. Teale KF, Devine A, Stewart H, Harper NJ. The management of metformin overdose. Anaesthesia. 1998;53(7):698-701.

12. Chang CT, Chen YC, Fang JT, Huang CC. High anion gap metabolic acidosis in suicide: don't forget metformin intoxication - two patients' experiences. Ren Fail. 2002;24(5):671-675.

13. Jagia M, Taqi S, Hanafi M. Metformin poisoning: A complex presentation. Indian J Anaesth. 2011;55(2):190-192. 